International Journal of Translation \& Community Medicine (IJTCM)

ISSN 2333-8385

\title{
Early Intervention and Prevention of Self-Injurious Behavior Exhibited by Children with Autism Spectrum Disorders (ASD)
}

Research Article

Shekhar, $\mathrm{C}^{1^{*}} \&$ Ganaie, S.A2 .

${ }^{1}$ Assistant Professor, Department of Psychology, University of Jammu, J\&K, India.

${ }^{2}$ Contractual lecturer, Department of psychology, University of Kashmir Hazratbal, J\&K, India.

\section{Abstract}

Autism is a Neuro-Developmental Disorder affecting socialization, communication with stereotyped and repetitive behaviors. The research Scientists all over world found that Self-injurious behavior (SIB) is a serious, chronic problem influencing about $10 \%$ to $14 \%$ of children with Autism Spectrum Disorders. (Iwata \& Rodgers, 1992). It occurs frequently in form of face slapping, head banging, and hand biting (Durand \& Crimmins, 1988). Such aberrant behavior is so obvious and physically harmful that catches caregivers' and special educators' attention all the times in naturalistic settings (Durand \& Crimmins, 1988). Injuries and health problems caused by this behavior could highly increase social isolation, restricted educational and vocational opportunities and costly medical and residential care (Kurtz et al., 2003). Therefore, early and appropriate intervention to decelerate such aberrant behavior is indeed needed. The purposes of this study were to assess the functions of self-injurious behavior in children with Autism Spectrum Disorders (ASD) and to explore early interventions to reduce such aberrant behavior.

Keywords: Autism Spectrum Disorders; Early Intervention; Self Injurious Behaviors Etc.

\section{*Corresponding Author:}

Chandra Shekhar,

Assistant Professor, Department of Psychology, University of Jammu, Jammu and Kashmir, India.

E-mail: dr.cschandra@gmail.com

Received: June 01, 2014

Accepted: July 08, 2014

Published: July 22, 2014

Citation: Shekhar, C. \& Ganaie, S.A. (2014). Early Intervention and Prevention of Self-Injurious Behavior Exhibited by Children with Autism Spectrum Disorders (ASD), Int J Translation Community Dis, 02(05), 42-45. doi: http://dx.doi.org/10.19070/2333-8385-140008

Copyright: Shekhar $\mathbf{C}^{\circ}$ 2014. This is an open-access article distributed under the terms of the Creative Commons Attribution License, which permits unrestricted use, distribution and reproduction in any medium, provided the original author and source are credited.

\section{Introduction}

Autism spectrum disorders (ASDs) are a group of developmental disabilities characterized by impairments in social interaction and communication and by restricted, repetitive, and stereotyped patterns of behavior. Symptoms typically are apparent before age 3 years. A person with Autism spectrum disorders (ASDs) may have a narrow range of interests that involve restricted behaviors and the tendency to perseverate on a single topic or activity. Patterns of repetitive and restrictive behaviors, described as stereotypes, often develop that serve multiple functions for a child (Heflin \& Alaimo, 2007). Self injurious behaviors (SIB) are common in children with ASD according to research by Baghdadli, Pascal, Grisi, and Aussilloux (2003). Their findings show that $50 \%$ of children in the study experienced Self Injurious Behavior, with $14.6 \%$ at severe levels. These behaviors can have far-reaching consequences and may restrict children from reaching their full potential.
There has been increased concern in developing successful interventions for problem behaviors in Autism spectrum disorders (ASDs) due to a rise in diagnoses of this disorder (Doughty \& Doughty, 2008).

Self injury is defined as repeated, self-inflicted, non accidental injury, producing bruising, bleeding or other temporary or permanent tissue damage. Self-injurious behavior (SIB) is defined as an act directed towards oneself that results in tissue damage (Tate $\&$ Baroff 1966). Self-injurious behaviors can also be defined as "actions that cause or have the potential to cause redness, bruising, or other injury to the body". A common SIB is skin picking, defined as "the scratching or picking of healthy skin...often producing tissue damage, infection, and potential scarring" (Ladd, Luiselli, \& Baker, 2009, p. 55). Self Injurious Behviour especially if continues over a longer period of time has proven to be extremely persistent and difficult to treat (Altmeyer et al.,) Self Injurious Behavior are often placed on a continuum with repetitive stereotypes, and have been suggested to only differ at the moment of injury (Jones, 1987). In fact, recent reviews of repetitive behaviors in autism place Self Injurious Behavior within its broad class (Turner, 1999). Self-injurious behaviors can range from severe, life-threatening injuries to less directly damaging cases. Repetitive behaviors may cause significant impairments to children with Autism spectrum disorders (ASDs) that can consume their waking hours and interfere profoundly with daily life. Some behaviors are considered socially inappropriate and may even become stigmatizing to a child. Self Injurious Behavior in particular can have short and long term damaging effects on a child and impact the entire family. The most serious effects are tissue damage, disfigurement, health risks due to infection, and stigmatizing consequences (Humenik, Curran, Luiselli, \& Child, 2008).

In 2003 a study was conducted in order to identify the risk factors for Self Injurious Behavior among children with Autism spectrum disorder. The results showed a correlation between Self 
Injurious Behavior and lower chronological age, higher degree of Autism spectrum disorder, and lower daily living skills. These variables may be helpful in predicting the occurrence of Self Injurious Behavior. This may also reinforce the idea that maladaptive behaviors raise the risk of Self Injurious Behavior in children with Autism spectrum disorders (ASDs) (Baghadadli et al., 2003).

There are surprisingly few empirical studies on emergence, early intervention or prevention of Self Injurious Behavior, and many of those studies are discussion articles or descriptive studies of common topographies, incidence or prevalence of Self Injurious Behavior. The remainder of this manuscript will focus on the emergence, early intervention and prevention of Self Injurious Behavior by covering three broad areas pertinent to Self Injurious Behavior: Causes, Functions and early intervention and prevention strategies.

\section{Self Injurious Behavior - Causes}

Several reasons are attributed for a person who indulges in selfinjurious behavior as it ranges from biochemical to social environment. The current paper will throw light on not just the causes of self injury but will equally dwell on describing the interventions:-

\section{Biochemical}

Researchers are of the belief that self-injurious behavior can be attributed to the levels of certain neurotransmitters. Scientifically speaking, beta-endorphins are nothing but endogenous opiatelike substances in brain and due to the effect of self injury, there can be increase in production along with release of endorphins. Hence, the sudden effect can make an individual anesthetic or worse of all; he/she ceases to feel pain while resorting to the behavior. There can also be another feeling which an individual can experience and that is the feeling of euphoria as it is primarily attributed with the release of hormones.

\section{Seizures}

The frontal as well as temporal lobes also become a prime seizure activity due to self-injurious behavior. One can usually associate the following activities such as biting of hand, banging of head, hitting chain, either slapping of ear and/or head and in few exceptional cases there can also be contact of knee to face. Owing to the fact that this is an involuntary behavior, the concerned individual needs to have self-restraint. For example, his/her arms should be tied for some time when he/she will have SIB etc.

\section{Arousal}

The arousal level of a person also has its association with selfinjurious behavior. It has been proved in the research that the arousal level of a person might be increased or decreased due to self-injury.

As per the under-arousal theory, few of the individuals who have low level of arousal indulge in self-injury in order increase the arousal level. This is the typical case where self-injury is attributed as an enhanced form of self-stimulation.

However, the over-arousal theory works totally opposite where it says that few individual works at an extremely enhanced or high level of arousal but resort to self arousal only to experience reduction in the arousal level. Hence, this is the practice which is adopted for the release of anxiety and tensions.

The reason attributed for high arousal level is psychological dysfunction and it may be enhanced due to stimulating environment. Hence as the arousal is reduced, the client may be tempted to work on inflicting injury to him/her especially at the time of encountering arousal-producing stimuli.

\section{Sensory}

Scratching as well as rubbing also comes in the purview of selfstimulation. Hence, in order to attain the highest degree of stimulation, a person damages the skin in the process as well.

\section{Frustration}

Frustration also results in creating death knell for a child by inflicting self injury. Even the modal which was made by Dollar and Company in the year 1939 is a testimony to the same. Person who lacks good communication skills often becomes the subject of frustration since he/she can't grasp the things which are being said to him/her.

\section{Communication}

The problems which are associated with communication often become the subject of self-injurious behavior. Since, a person who can't express in a proper manner automatically gets into frustration and is thus prone to self-injury.

\section{Social Attention}

It has been proved from various researches that positive attention has the tendency to greatly increase the frequency of positive reenforcement while if the behavior is ignored then it only leads to decrease in frequency which in other way implies extinction.

\section{Obtain Tangibles}

Individual in their unsuccessful attempt of obtaining an object might also engage in self injurious behavior. In the event when the child receives the object, then it is termed as positive behavior through tangibility.

\section{Avoidance/Escape}

In order to avoid social interaction, individuals might resort to self injury. For example, in the event when the client is being told by the caretaker to do certain activities and if he/she refuses to comply, then in order to avoid the same he/she indulges in selfinjury. As a result, the whole action is then directed towards the action of client.

\section{Self Injurious Behavior- Functions}

Self injurers get immense pleasure as well as relief from emotional trauma which is hovering around them.

i. It equally gives a soothing effect in countering as well as managing intolerable feelings.

ii. It also ensures increasingly high level of security thanks to external soothing. It in a way is termed to be having a regulating effect as well.

iii. It gives way to creating a pain of its own, by mastering over 
the pain later on. Hence, it avoids being victim of the same.

iv. It equally gives them a great level of courage and zeal to master the earlier feelings which are yet to be resolved.

v. The gestures as well as behavior gives arms and ammunition as a form of defense to the injurer from the usual and most obvious emotion of being abandoned altogether. The injurer gets to know his own state of mind as well as present condition by viewing blood and scars which was the result of physical sensation. The wounds in the body act as a source of connection to the individual with his/her both inner as well as outer world. The whole sequence of events in terms of control as well as mastery stands as a witness to optimism and hope.

\section{Early Intervention \& Prevention for CwASDs Frequency of self-injury}

In order to start the whole process of intervention, one needs to be familiar and knowledgeable about the current frequency with which the behavior occurs in order to gauge the effectiveness of the intervention. The concerned person, who is looking after the child, should observe self-injury. This practice equally helps in minutely detecting even days as well as times when self injury has been quite frequent and common. Upon starting the intervention, records as well as reviews need to be placed in order to gauge the working status of intervention.

\section{Child's safety holds paramount importance}

In the process of reducing self-injury's frequency, a child's safety can't be compromised. Therefore, one needs to minutely observe how the behavior responds.

For an effective intervention to take place, at the time of selfinjury, one needs to take care about the whole action that is not reinforcing. Like in the event when the child is banging his/her head and can potentially damage his head or face, then handle the whole situation without making eye contacts and it is termed to be a reinforcing act.

\section{Problems associated while reducing the reinforce- ment}

Others can't reinforce self injury and it although being a great way to reduce self-injury but it is accompanied with problems. In order to ensure its effectiveness, self injury needs to be conducted upon its demonstration. Everyone should be familiar with the ways which trigger self-injury on a child and the steps to avoid its reinforcement. Or else when the person fails to take note of the reactions it did in the past, then it will potentially provoke the response (which is given the name of extinction burst). Here, reinforcement will pave the way for accolades and rewards of even deadlier and severe form. Hence, there is a dire need to limit the number of reinforcement without compromising on the safety of child. You should also use the following other methods in order to combine the reduction of rewards

\section{Using comparably more adaptive behavior for re- placing self-injury}

Using a bit more adaptive behavior in place of self injury is indeed an intervention of sorts as it also primarily functions with reinforcing adaptive behavior and it holds traits like that of self- injury. Needless to say, that this is done for the child's safety. In order for the whole process to be $100 \%$ effective, steps should be taken to identity the consequences and antecedents of self-injury by taking care of an equivalent behavior. Since, this type of behavior comprises the similar functions like that of self-injury.

\section{Functional communication Training}

This training is highly beneficial amongst children who are suffering with various intellectual disabilities. Here, as a child being alone, is taught to communicate for the purpose of attention. Here, one needs to understand that the behavior comparably gains greater degree of significance than self-injury for attaining a quick as well as fast response.

\section{Time when reinforcement is to be given}

Reinforcing behavior is indeed quite an effective and great way to reduce self-injury. There are various principles which can indeed be applied in a successful manner. Like Differential Reinforcement of other behavior (DRO) assists in providing positive reinforcement at the time especially when there is no display of positive reinforcement for a greater time period. Differentia Reinforcement of Incompatible behaviors (DRI) works towards eliminating self-injury. Since, it reinforces other behaviors which don't show any compatibility with self injury. Non Contingent Reinforcement (NCR) is another technique which provides reinforcement irrespective of how self-injury is presented.

\section{Making the tasks functional and compatible}

In the event when the task is meant to be reinforcing self-injury, then during such times, it is best to reduce the aversiveness of task as it stands out to be an important thing to reduce self-injury. In order to make the process effective, keep a note of frequency of self injury after every task and compare their nature at the end. The task seems to be more aversive if there is a higher frequency of self-injury. In the event when it is difficult to gauge, then systematically modify it by changing the elements. Since, when the task is less aversive then self-injury decreases. It is also important to simplify the task and it happens when you break it down thus enabling the child to stick with one act at a time.

\section{Protective devices}

In the event when the self injury is quite severe, then one should not hesitate in wearing the protective devices. It protects the parts from permanent injury especially at the time when self-injury is quite severe. However in the process, restraints should be used in the following manner: 1]. Professional physiotherapist needs to provide 2]. Reviews is done constantly in order to gauge the chance for its removal 3]. Clinical Psychologist devises the part of programme 4]. The overall over time is reduced due to planned programme. It can be understood in the form of an illustration where as a child wears an arm splint is restricted to hit his/her head.

\section{Applied Behavior Analysis (ABA)}

Applied behavior analysis (ABA) is the process of applying interventions that are based on the principles of learning. ABA methods are used to increase and maintain desirable adaptive behaviors, reduce interfering maladaptive behaviors, develop new skills 
and generalize behaviors to new environments or situations.

Positive Behavioral Support (PBS) is an approach that tries to increase positive behaviors, decrease problem behaviour and improve the child's or adult's lifestyle. The PBS method focuses on the interactions between people with autism, their environment, their behaviour and learning processes to develop the best lifestyle for them.

\section{Educational Intervention}

Education has been defined as the fostering of acquisition of skills and knowledge to assist a child to develop independence and personal responsibility. It encompasses not only academic learning but also socialization, adaptive skills, communication, amelioration of interfering behaviors and generalization of abilities across multiple environments.

Individualized Education Programmes (IEPs) properly designed and systematically practiced are an effective way to deal and prevent problem behaviors such as self injurious behaviors etc. and help to learn appropriate adaptive skills.

\section{Social Skills Training}

Social skills are necessary to be a competent adult in any society. Joint attention training may be especially beneficial in young, preverbal children with ASDs, because joint attention behaviors precede and predict social language development.

Social skills training focuses on child's responding to the social overtures of other children and adults, initiating social behavior, and minimizing stereotyped behaviors while using a flexible and varied repertoire of responses and self-managing new and established skills.

A social skills curriculum should target social skills groups, social stories, visual cueing, social games, video modeling, scripts, peer-mediated techniques. Play and leisure curriculum supported primarily by descriptive and anecdotal literature are some of the methods through which the social skills of children with autism can be improved. Social Skills training is always helpful in reducing problem behaviors.

\section{Conclusion}

There are various research studies which show many options of early interventions for children with Autism spectrum disorders (ASDs) who experience Self Injurious Behavior. But firstly we have to conduct the functional behavior assessment of children with Autism spectrum disorders (ASDs) in order to form a hypothesis of the function of the behaviors. A behavior analysis will disclose the relationship between the Self Injurious Behavior and the antecedents and consequences of the behavior. Then an intervention plan can be developed that is specific for the individual. Various researches on early interventions for Self Injurious Behavior include some reinforcement strategies, along with other methods to reduce the behaviors. But the reality is that there is no single intervention to prevent or decrease Self Injurious Behavior in children with Autism spectrum disorders (ASDs) due to the individualized characteristics of the child and the behavior. Autism spectrum disorders (ASDs) presents in a broad spectrum of features and Self Injurious Behavior can present itself in many different aspects. The key is to base the intervention on the results of the functional behavior assessments, when the functions of the behaviors are known.

There is need for future research to identify possible early interventions for children who exhibit Self Injurious Behavior. Early intervention could make a big difference in the ability of these children and lessen the effect that these behaviors can have on their life.

\section{Acknowledgements}

We thank to Research Scholar Ms. Tamanaah, Ganie Zahoor, Ganie Parveez, Ganie Khursheed and Prof. S.S.Bali (Aasra Jammu $\mathrm{J} \& \mathrm{~K})$.

\section{References}

[1]. Bachman J.A (1972) Self injurious behavior: A behavioral analysis. Journal of Abnormal psychology 80:211-244.

[2]. Carr E.G (1977) The Motivation of self Injurious Behavior: A review of some hypotheses. Psychological Bulletin 84:800 - 816 .

[3]. Baghdadli A, Pascal C, Grisi S, Aussilloux C (2003) Risk factors for selfinjurious behaviours among 222 young children with autistic disorders. Journal of Intellectual Disability Research 47:622-627.

[4]. Doughty S.S, Doughty A.H (2008) Evaluation of body-pressure intervention for self-injury in autism. Behavioral Development Bulletin 14:23-29.

[5]. Heflin L.J, Alaimo D.F (2007) Students with autism spectrum disorders: Effective instructional practices. Columbus, $\mathrm{OH}$ : Pearson.

[6]. Mahatmya D, Zobel A, Valdovinos M.G (2008) Treatment approaches for self-injurious behavior in individuals with autism: Behavioral and pharmacological methods. Journal of Early and Intensive Behavior Intervention 5:106-118.

[7]. Soares D.A, Vannest K.J, Harrison J (2009) Computer aided self-monitoring to increase academic production and reduce self-injurious behavior in a child with autism. Behavioral Interventions 24:171-183.

[8]. Hall S, Oliver C, Murphy G (2001) Early development of self-injurious behaviour: An empirical study. American Journal on Mental Retardation 106(2):189-199.

[9]. Murphy G, Wilson B (1985) Self injurious Behaviour. Kidderminster: British Institute of Mental Handicap Publications.

[10]. Durand V. M, Crimmins D. B (1988) Identifying the variables maintaining self injurious behvaior. Journal of Autism and Developmental Disorders 18:99-117.

[11]. Allen C (1995) Helping with deliberate self-harm: Some practical guidelines. Journal of Mental Health 4:243-248. 